\title{
CAPNetz - Kompetenznetzwerk ambulant erworbene Pneumonie: Strukturen und Ziele
}

\author{
T. Welte \\ R. Marre \\ N. Suttorp
}

\author{
CAPNet-Network of Competence for Community Acquired Pneumonia: \\ Structures and Aims
}

\section{Zusammenfassung}

Die ambulant erworbene Pneumonie (Community Acquired Pneumonia/CAP) ist auch in Deutschland eine Erkrankung mit hoher Morbidität und Mortalität. Trotz der Bedeutung der CAP fehlen hier zu Lande zuverlässige Daten zum Erregerspektrum, zur Resistenzsituation der Erreger, zum Verlauf der Erkrankung ebenso wie evidenzbasierte Therapieempfehlungen. Auch die Grundlagenforschung in diesem Bereich ist stark verbesserungswürdig. Das Bundesministerium für Bildung und Forschung (BMBF) hat diese Defizite erkannt und fördert deshalb seit 2002 CAPNetz, das Kompetenznetzwerk ambulant erworbene Pneumonie.

\section{Abstract}

Community Acquired Pneumonia (CAP) is a disease with high morbidity and mortality in Germany. Apart from the importance of CAP, no reliably data is available about infective germs, resistance of germs, course of the disease, or evidence based treatment. Also basic research should be improved in this field. The German State Ministry for Research (BMBF) has recognised these deficits and supports CAPNet, the network of competence for Community Acquired Pneumonia since 2002.

\section{CAP: Definition und klinische Symptome}

Eine Infektion des Alveolarraums, die zur Akkumulation von Entzündungszellen und von Sekret in den Alveolen und zu einer Störung des Gasaustauschs führt, wird als Pneumonie bezeichnet [1].

Frühere Klassifikationen orientierten sich wechselseitig an pathologischen (Lobär- oder Bronchopneumonie) oder mikrobiologischen [2] (typische - Pneumokokken, Staphylokokken oder gram-negative Keime - oder atypische - Chlamydien, Mykoplasmen, Legionellen - Erreger) Unterschieden einzelner Pneumo- nieformen. Eine Reihe von Studien konnte zeigen, dass weder die Differenzierung zwischen typisch und atypisch [3] noch eine an der morphologischen Veränderung orientierte Einteilung [4] verwertbare Hinweise für die Therapie dieser Infektion liefern können.

Die Unterscheidung in ambulant erworbene oder im Krankenhaus entstandene Pneumonien hat sich heute aufgrund der Konsequenzen dieser Einteilung für das diagnostische und therapeutische Vorgehen etabliert [5]. Als dritte Gruppe werden Pneumonien bei immunsupprimierten Patienten (nach Chemotherapie oder Transplantation bzw. bei Patienten mit Systemerkrankung 
oder AIDS) unterschieden, da hier opportunistische Erreger (Pneumocystis carinii, Pilze, Viren) im Vordergrund stehen [6]. Die empirische Primärtherapie ist entsprechend unterschiedlich.

Jede Pneumonie, bei der der auslösende Erreger außerhalb des Krankenhauses aufgenommen wurde, wird als ambulant erworben bezeichnet [5]. Unter nosokomialer Pneumonie wird dagegen jede Infektion verstanden, die 48 Stunden oder später nach Krankenhausaufnahme oder in den ersten 14 Tagen nach Krankenhausentlassung auftritt [7].

Schon bei der Definition der ambulant erworbenen Pneumonie gibt es erhebliche Differenzen zwischen einzelnen Fachgesellschaften. Rein formal beweisend für das Vorliegen der Erkrankung wäre der pathologisch-histologische Befund, verbunden mit einem positiven Keimnachweis im Gewebe. Das ist jedoch realitätsfern, da Biopsiematerial in der Regel von Pneumoniepatienten nicht erhältlich ist.

Ein anderes Vorgehen wählte die European Respiratory Society, die auf ein durch klinische Zeichen definiertes „untere Atemweginfektion" (lower respiratory tract infection - LRTI) benanntes Krankheitsbild baut, das sowohl die eitrige Tracheobronchitis als auch die Pneumonie erfasst [8].

Im angloamerikanischen Sprachraum wird auch das Vorhandensein eines neuen oder progressiven Infiltrats im Röntgenbild als conditio sine qua non der Pneumoniediagnose angesehen, klinische Befunde (Auskultationsbefund, Fieber, Husten und Auswurf, Dyspnoe, Thoraxschmerz und Entzündungszeichen im Serum) werden als Hilfskriterien in Anspruch genommen [9]. Im Rahmen des CAPNetz haben wir uns - auch aus Gründen der Vergleichbarkeit mit internationalen Studienergebnissen - dafür entschieden, dem Röntgenbild eine zentrale Bedeutung zuzuerkennen. Die Organisation und Bezahlung dieser Untersuchung im ambulanten Bereich wird daher vom Netzwerk übernommen (Abb.1).

\section{Datenlage rund um CAP unbefriedigend Epidemiologie}

Pneumonien sind weltweit eine ernsthafte Bedrohung für die Gesundheit und ein enormes sozio-ökonomisches Problem für die Gesundheitssysteme. Nach Angaben der WHO versterben jährlich etwa 3-4 Millionen Menschen, insbesondere im Kindesund Greisenalter, durch Pneumonien. Damit steht Pneumonie in der Todesursachenstatistik weltweit an dritter Stelle bei den Infektionskrankheiten [10].

Exakte epidemiologische Daten liegen aus den USA vor. Hier führen 2-3 Millionen CAP-Fälle pro Jahr zu etwa 10 Millionen Arztkontakten [11]. Schätzungsweise 20\% ( $1 / 2$ Million) dieser Patienten werden stationär aufgenommen, die Inzidenz liegt bei 258 Hospitalisationen pro 100000 Einwohner. Die Notwendigkeit der Krankenhausbehandlung ist altersabhängig, die höchsten Raten finden sich bei Patienten $>65$ Jahre, bei denen die Zahl der Krankenhausaufnahmen um das Vierfache auf annähernd 1000 pro 100000 Einwohner ansteigt [12]. Insgesamt wird für die USA mit 8 Milliarden \$ Kosten für die Pneumoniebehandlung gerechnet, die in der Mehrzahl auf ältere und hospitalisierte Patienten entfallen (Tab.1).

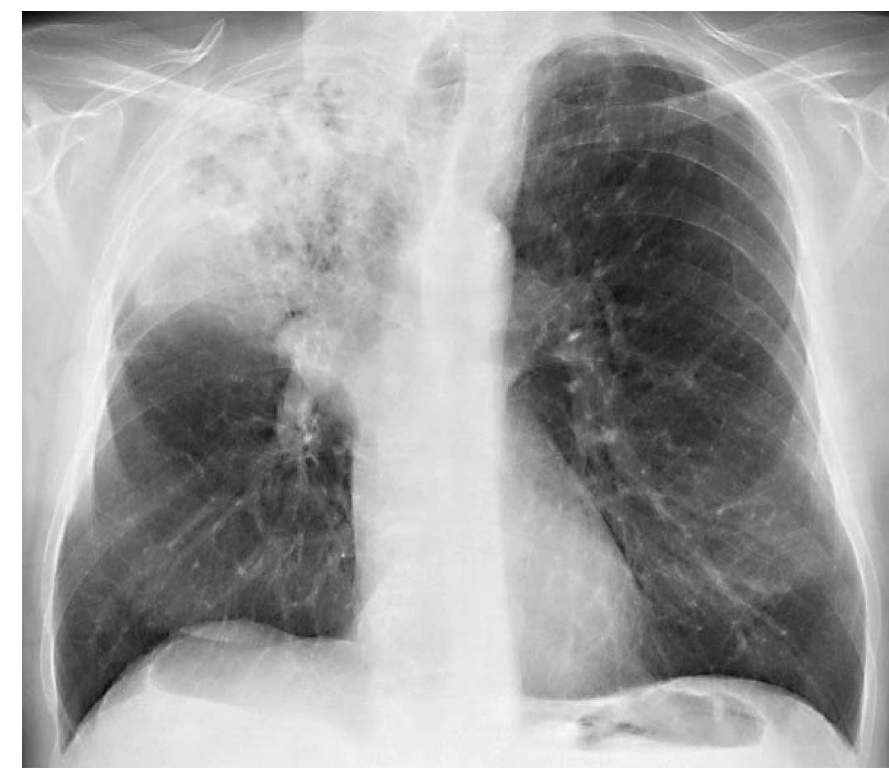

Abb. 1 Lobärpneumonie.

Tab. 1 Kosten für die Pneumoniebehandlung (1998)

\begin{tabular}{lllll}
\hline $\begin{array}{l}\text { Alter } \\
\text { (Jahre) }\end{array}$ & $\begin{array}{l}\text { Kosten im ambu- } \\
\text { lanten Sektor }\end{array}$ & $\begin{array}{l}\text { Kranken- } \\
\text { hauskosten }\end{array}$ & $\begin{array}{l}\text { Pflege- } \\
\text { dienst }\end{array}$ & $\begin{array}{l}\text { Summe } \\
\text { (Mill. \$) }\end{array}$ \\
\hline$\rightarrow 65$ & 119 & 4412 & 305 & 4836 \\
$\leftarrow 65$ & 266 & 3139 & 192 & 3597 \\
\hline alle & 384 & 7552 & 497 & 8433 \\
\hline
\end{tabular}

Auch in Deutschland hat die CAP schon heute den Stellenwert einer „Volkskrankheit“.

Nach Angaben des Statistischen Bundesamtes mussten im Jahre 1998240000 Patienten wegen CAP in ein Krankenhaus aufgenommen werde. Damit führt die CAP häufiger zur stationären Aufnahme als die bekannten Volkskrankheiten Myokardinfarkt und Schlaganfall.

Da erfahrungsgemäß jeder vierte bis fünfte CAP-Patient stationär behandelt werden muss, kann man in Deutschland von ca. 800000 Patienten mit CAP pro Jahr ausgehen. Die Annahme, dass mit der zunehmenden Überalterung der Bevölkerung die Inzidenz der CAP in den nächsten Jahren weiter deutlich zunehmen wird, ist sicherlich korrekt.

Auch wenn die Pneumoniemortalität von 1970 bis 1980 zurückgegangen ist und sich heute auf dem Stand von vor 20 Jahren bewegt, liegt sie immer noch bei $6-8 \%$. Sie ist damit die sechsthäufigste Todesursache in Deutschland. Vor allem ältere Personen sind gefährdet. Zahlen aus dem Jahr 1996 weisen aus, dass etwa $70 \%$ der an Pneumonie Verstorbenen Männer waren und dass fast $90 \%$ der Frauen, die an Pneumonie verstarben, älter als 75 Jahre waren (Tab. 2) [13].

Allerdings tragen Pneumonien auch bei der Gruppe der jüngeren Patienten erheblich zur Arbeitsunfähigkeit bei. Nach Angaben 
Tab. 2 Krankenhausaufnahmen in Deutschland 1998 (Quelle: Statistisches Bundesamt)

\begin{tabular}{ll}
\hline Erkankung & Krankenhausaufnahmen $\mathbf{1 9 9 8}$ \\
\hline ambulant erworbene Pneumonie & 238659 \\
\hline Sepsis & 17949 \\
\hline Tuberkulose & 12904 \\
\hline Virushepatitis & 10927 \\
\hline Meningitis & 2837 \\
\hline Aids & 5448 \\
\hline arterielle Hypertension & 185083 \\
\hline Schlaganfall & 161758 \\
\hline Myokardinfarkt & 132501 \\
\hline
\end{tabular}

der Statistik der Ortskrankenkassen wurden alleine $1996 \mathrm{mehr}$ als 1,23 Millionen Arbeitsunfähigkeitstage registriert.

\section{Diagnostik und Therapie}

Wie oben gezeigt, entfallen 70\% der Kosten der Pneumoniebehandlung auf die Versorgung stationär behandelter Patienten. Neuere Untersuchungen zeigen, dass eine bessere Selektion der Patienten anhand der von Fine publizierten Schweregradkriterien [14] zu einem Rückgang der Hospitalisationsrate um 15-20\% führen könnte [15].

Andererseits zeigt sich eine weiterhin nennenswerte Letalität von Pneumoniepatienten im Krankenhaus. Hauptgründe hierfür sind die verzögerte Einweisung von Patienten ins Krankenhaus und eine späte und falsche primäre Antibiotikatherapie [16]. Nationale und internationale Pneumonie-Management-Richtlinien haben sich im deutschen Praxisalltag bisher kaum etablieren lassen, obwohl für USA gezeigt wurde, dass solche Empfehlungen Letalität und Krankheitskosten günstig beeinflussen können [17].

Obwohl sich bisher keine Steigerung der Letalität durch resistente Erreger nachweisen lässt [18], wächst die Besorgnis über die weltweit zunehmende Unempfindlichkeit der wichtigsten Erreger gegen die gängigen Antibiotika.
Dabei existieren jedoch gravierende Unterschiede zwischen einzelnen Ländern. Die für Deutschland vorliegenden Zahlen des nationalen Referenzzentrums für Streptokokken [19] auf der Basis von Daten aus 19 mikrobiologischen Labors bestätigt eher niedrige Resistenzraten von Pneumokokken gegenüber Penicillin G, die $3 \%$ auf keinen Fall übersteigen. Die Zahl der Sputumproben ist allerdings in allen Untersuchungen gering. Insgesamt handelt es sich um selektive Daten, von denen wir nicht wissen, ob sie die Infektionsepidemiologie in der Praxis auch nur annähernd widerspiegeln. Eine breit angelegte Untersuchung in Allgemeinarztpraxen in Großbritanien [20] lässt jedenfalls vermuten, dass sich der ambulante Bereich erheblich vom stationären unterscheidet. Bemerkenswert ist der Anstieg der Makrolidresistenz von Pneumokokken auf $10-15 \%$.

Es ist aber zu früh, deshalb die Stellung der Makrolide, die in allen gängigen Empfehlungen als First-Line-Antibiotikum geführt werden, zu ändern. In neueren Empfehlungen der Nordamerikaner werden neben Makroliden und anderen Antibiotika auch neuere Fluorchinolone genannt, obwohl für Letztere kürzlich bereits erste Resistenzen beschrieben wurden. [21]. Besonders problematisch ist dabei, dass sich die Zahl resistenter Erreger mit zunehmendem Alter von Pneumoniepatienten erhöht. Als besonderes Problem erweist sich dabei, dass sich das Erregerspektrum mit zunehmendem Alter ändert. Atypischen Erregern wie Chlamydien und Legionellen kommt vermutlich eine besondere Bedeutung zu. [22].

Dieser Mangel an infektionsepidemiologischem Wissen und konkreter Evaluierung diagnostischer und therapeutischer Vorgaben für die Situation in Deutschland hat zur Entstehung der CAPNetz-Initiative beigetragen.

\section{Ziele des interdisziplinären Netzwerkes}

Hauptaufgabe von CAPNetz ist es, Fachwissen zur ambulant erworbenen Pneumonie aus den verschiedensten medizinischen Bereichen zusammenzutragen, die unterschiedlichen Gruppen zu vernetzen, Synergien zu fördern, deutschlandspezifische Daten zu ermitteln und so ein bundesweites Netzwerk von höchster Kompetenz mit einer ausgewiesenen Expertise zu schaffen.

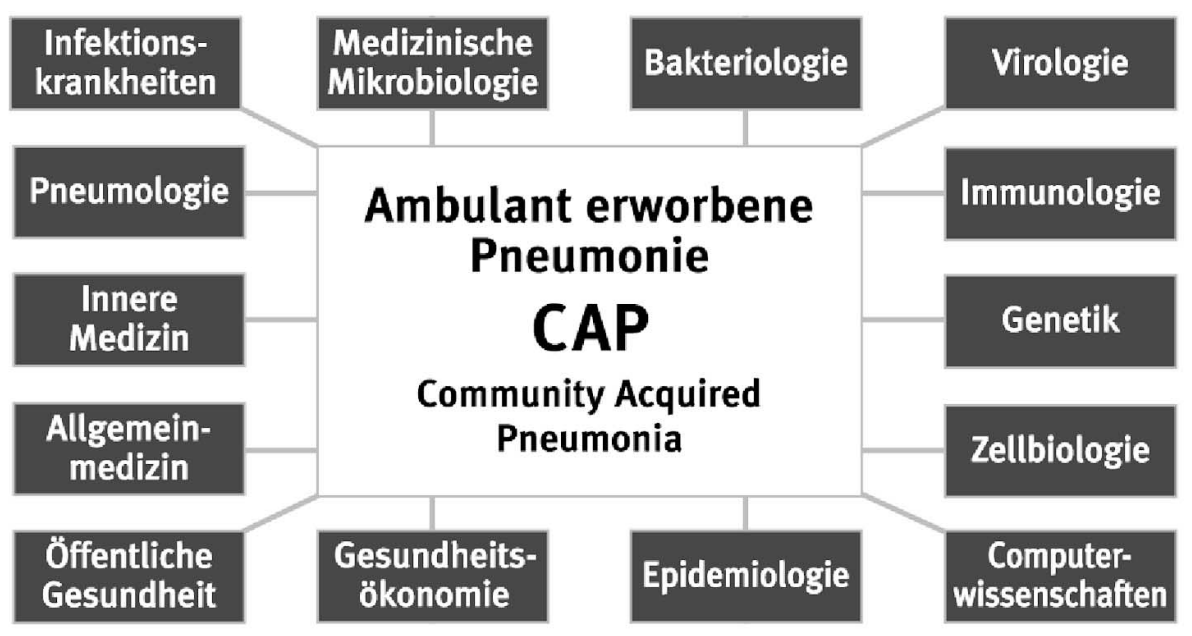

Abb. 2 Das CAPNetzwerk. 
Hierbei werden niedergelassene Ärzte Krankenhausärzte, Mikrobiologen, Virologen, Epidemiologen, Computerexperten und ITSpezialisten in enger Kooperation zusammenarbeiten. Alle klinischen und mikrobiologischen Daten werden zusammengeführt und in einer zentralen Material- sowie Informationsdatenbank verwaltet (Abb. 2).

Die Ziele, die CAPNetz langfristig verfolgt, sollen die Versorgungsqualität von Patienten mit CAP erhöhen. Der Katalog der auf längere Sicht hin zu realisierenden Ziele umfasst zehn Vorhaben:

- Verbesserung der Diagnostik durch Zusammenführung und Interpretation von klinischen und mikrobiologischen Daten

- Verbesserung der Therapie durch Evaluierung von Therapieempfehlungen

- Förderung von Maßnahmen zur Einhaltung von Impfempfehlungen. Verbesserung der Prävention durch Evaluierung von Impfprogrammen

- Einrichtung von mikrobiologischen und genetischen Datenbanken zu den gewonnenen Erregern und Stämmen

- Identifizierung von „neuen“ bisher im Hinblick auf Pneumonie nicht erkannte Pathogenen

- Aufbau einer zentralen Datenbank zur antimikrobiellen Resistenz

- Aufbau einer zentralen Datenbank für die klinischen Daten

- Früherkennung von Hochrisikopatienten durch Charakterisierung von „toll-like“-Rezeptoren und TNF-Polymorphismus

- Verbesserung des Verständnisses über Keime und Erreger Wirt - Interaktion mit Bezug auf die bei CAP relevanten Erreger, Ziel ist eine Pneumonie-Therapie über Antibiotika hinaus zu entwickeln.

- Verbesserung der fachlichen und wissenschaftlichen Ausbildung auf allen Ebenen (Patienten, Ärzte, Grundlagenforscher)

\section{CAPNetz: Vernetzung in allen Ebenen}

Durch die finanzielle Unterstützung des Bundesministeriums für Bildung und Forschung werden die Zentren der Forschung und Krankenversorgung miteinander vernetzt („vertikale Vernetzung“) und Verbindungen zu niedergelassenen Ärzten und lokalen Projektgruppen sowie den Patienten geschaffen („horizontale Vernetzung“"). Die in den Schwerpunktzentren vorhandenen Forschungsmöglichkeiten, die Informationstechnologien des Netzwerkes sowie die Datenbanken können so von allen Netzteilnehmern genutzt werden.

Die meisten der vom BMBF geförderten Netzwerke beschäftigen sich mit Krankheiten,

- für die es nur eine sehr begrenzte Anzahl von Experten gibt,

- für die es spezialisierte Abteilungen an Kliniken der Maximalversorgung existieren und

- bei denen die Patienten in der Regel stationär behandelt werden.

Solche Voraussetzungen in der Infrastruktur erleichtern dementsprechend den Aufbau von Netzwerken. CAP ist im Gegensatz dazu eine Infektionskrankheit, die in erster Linie vom Hausarzt gesehen und behandelt wird. Nur ein Teil der Patienten bedürfen einer stationären Behandlung. Vor diesem Hintergrund bietet CAPNetz die einmalige Chance, Expertenwissen aus den unter- schiedlichsten Fachrichtungen und den unterschiedlichen medizinischen Versorgungsebenen zusammenzubringen und zu bündeln. Auch wird es interessant sein zu sehen, wie Allgemeinmediziner und Hausärzte für die Mitarbeit im Netzwerk motiviert werden können, wie effizient die Probenentnahme und -weiterleitung sowie der Datentransfer organisiert werden kann und wie gut der Aufbau von zentralen Datenbanken gelingt (Abb. 3).

\section{Acht klinische Zentren und 670 Netzwerkärzte}

Das Netzwerk umfasst derzeit acht bereits etablierte bzw. gerade im Aufbau befindliche lokale klinische Zentren in Berlin (Prof. Lode), Rotenburg/Wümme (Prof. Schaberg), Essen (Prof. Teschler), Köln/Bonn (PDs Drs. Ewig/Fätkenheuer), Lübeck (Prof. Dalhoff), Lüdenscheid (Prof. Lorenz), Magdeburg (PD Dr. Welte) und Würzburg (Prof. Jany). Diese repräsentieren Kliniken und Ambulanzen aller Versorgungsstufen, in denen Untersuchungen und Therapien im Rahmen der klinischen Pneumologie angeboten und durchgeführt werden können. Mit diesen lokalen Zentren kooperieren insgesamt etwa 670 niedergelassene Allgemeinärzte, Internisten und Pneumologen und mehr als 30 Krankenhäuser. Konsiliarlaboratorien für CAP-relevante Keime an den Universitäten Dresden und Jena sowie die Nationalen Referenzzentren für Influenza (Robert-Koch-Institut Berlin) bzw. Streptokokken (RWTH Aachen) begleiten und unterstützen die Arbeit von CAPNetz. Unterstützung aus der Wissenschaft kommt von der Deutschen Gesellschaft für Pneumologie (DGP), der European Respiratory Society (ERS), der Deutschen Gesellschaft für Infektiologie (DGI), der Deutschen Gesellschaft für Mikrobiologie und Hygiene (DGHM), der Deutschen Gesellschaft für Internistische Intensivmedizin sowie der Deutschen Gesellschaft für Innere Medizin (DGIM) (Abb. 4).

\section{Dreiergremium koordiniert die Arbeit}

Koordiniert wird die Arbeit von einem dreiköpfigen wissenschaftlichen Gremium: Professor Norbert Suttorp von der Medizinischen Klinik m.S. Infektiologie an der Charité als CAPNetz-
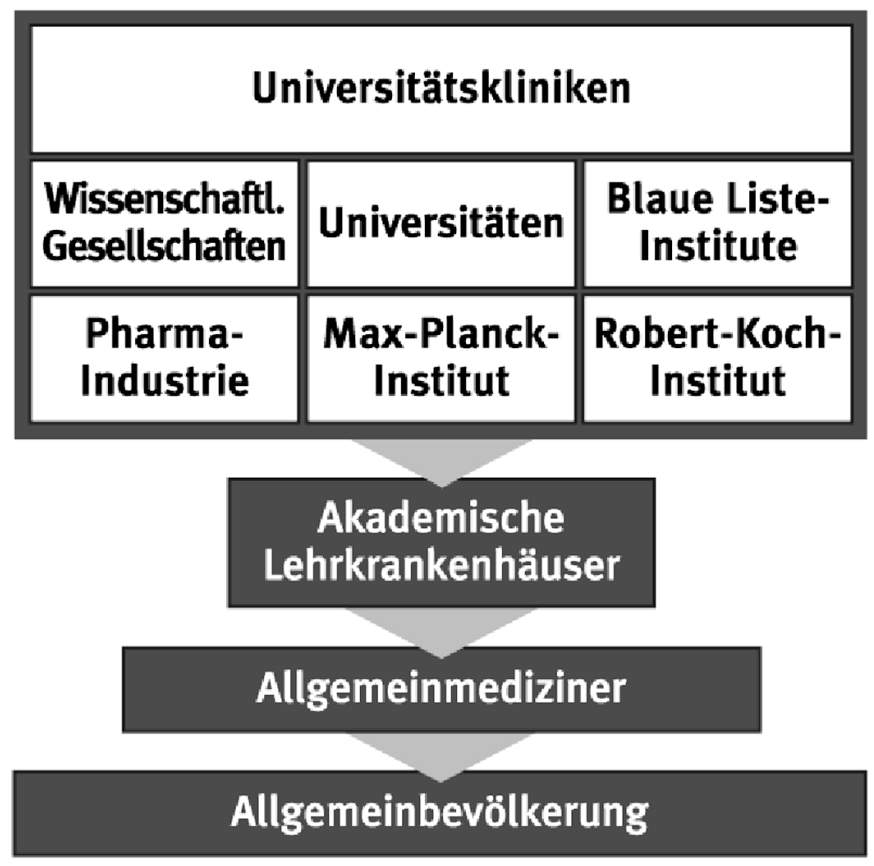

Abb. 3 Horizontale und vertikale Vernetzung in CAPNetz. 


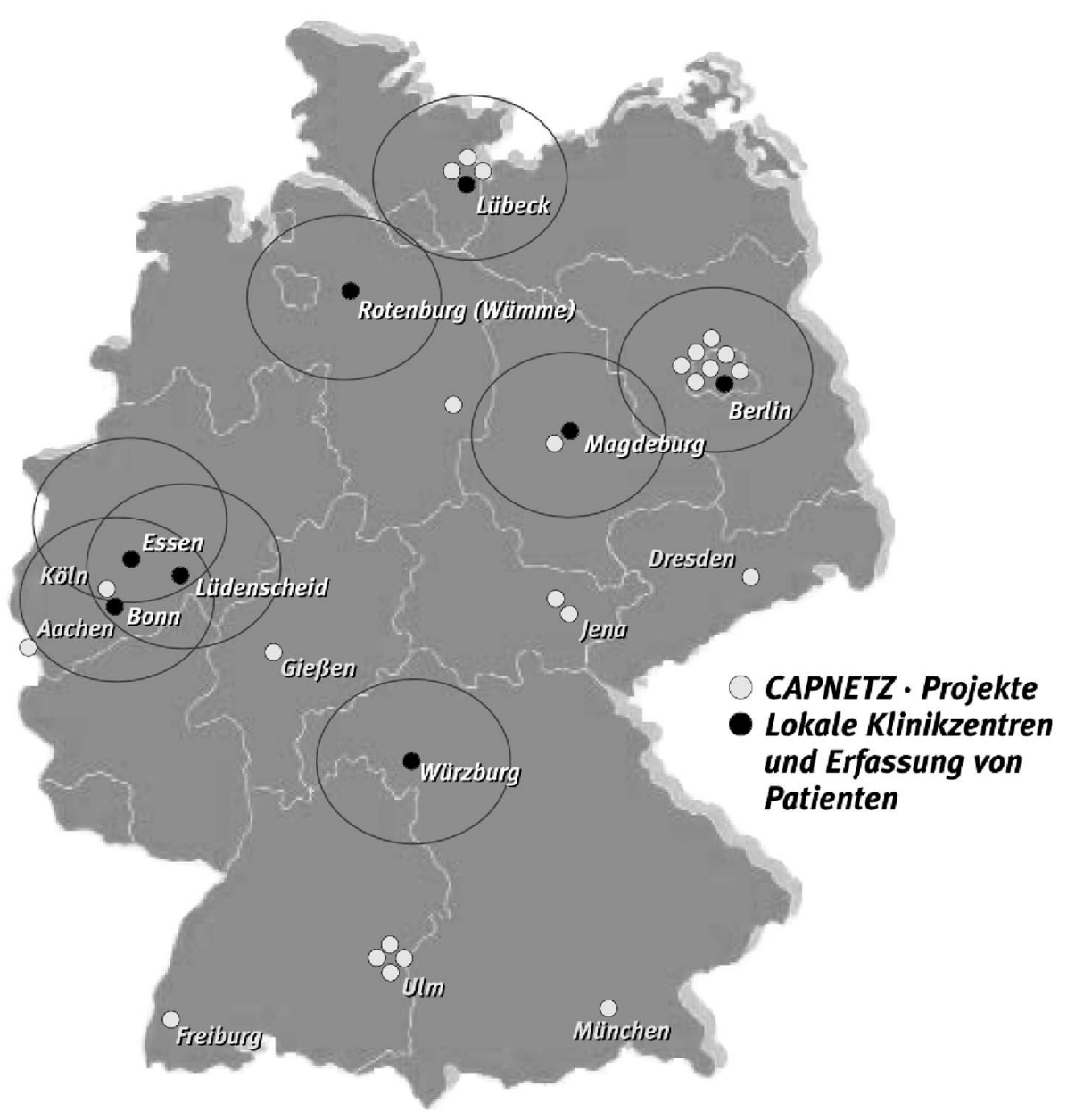

Abb. 4 Landkarte mit lokalen Zentren in Deutschland.

Sprecher, PD Dr. Tobias Welte von der Abteilung für Pneumologie an der Universität Magdeburg und von Professor Reinhard Marre von der Abteilung für Medizinische Mikrobiologie und Hygiene an der Universität Ulm. In Ulm befindet sich auch die Geschäftsstelle von CAPNetz, die von Dr. Klaus Richter geleitet wird und die als Zentrale Serviceeinheit die Bereiche Verwaltung, Qualitätssicherung, Aus- und Weiterbildung sowie Kommunikation bündelt.

\section{CAPNETZ: Wissenschaftliches Programm}

Eines der wesentlichen Elemente der Arbeit von CAPNetz wird die Durchführung von klinischen Studien auf höchstem Niveau und nach internationalen Standards sein. Eine elektronische Patientenrekrutierung via „remote data entry“ ist ein essenzielles Feature von CAPNetz, um state of the art-gemäß und ohne Papier die aufwändige Dokumentation zu gewährleisten. Ausgehend von dem generierten Datenmaterial werden u.a. folgende Fragestellungen untersucht werden:

- Wie häufig verursachen welche Erreger eine ambulant erworbene Pneumonie?

- Wie ist die Resistenzsituation der Erreger gegenüber Antibiotika in Deutschland?

- Gibt es im Blut Prädiktoren, die eine Vorhersage über Krankheitsschwere und -verlauf ermöglichen?

- Ist das z.Z. in Deutschland praktizierte Vorgehen bei ambulant erworbener Pneumonie ökonomisch?

- Müssen bestehende offizielle Empfehlungen zur Diagnose und Therapie der ambulant erworbenen Pneumonie überarbeitet werden?
- Wie kann eine bessere Akzeptanz von Impfungen erreicht werden?

- Was bringt Bakterien dazu, Schleimhäute nicht nur zu besiedeln, sondern invasiv zu werden?

- Warum kann das körpereigene Immunsystem die Erkrankung nicht verhindern?

Um valide Aussagen treffen zu können, ist der Zugang zu einer großen Zahl von Patienten notwendig. Nur ein großes Netzwerk kann ein solch umfangreiches Patientengut in kurzer Zeit erfolgreich generieren. Die lokalen Zentren sind über ganz Deutschland verteilt und werden innerhalb von nur drei Jahren etwa 6000 Patienten einschließen. Dabei ist davon auszugehen, dass in jeder angeschlossenen Praxis pro Jahr etwa 3 bis 5 Patienten mit CAP generiert werden. Die Zentren sind so verteilt, dass sie etwa $1 \%$ der jährlich in der Bundesrepublik auftretenden Fälle von CAP erreichen können.

Zur Durchführung begrenzter Forschungsvorhaben in einzelnen Projekten schreibt CAPNetz einjährige Stipendien aus. Die Stipendien können auch der Einführung in eine besondere Forschungsrichtung oder dem Erlernen bestimmter Methoden aus dem Themengebiet von CAPNetz dienen; sie können im In- und Ausland in Anspruch genommen werden.

\section{Bündelung der Forschungsaktivitäten in Projektbereiche}

Die wissenschaftlichen Forschungsaktivitäten sind in die Projektbereiche A bis D und Z gebündelt. 
Im Projektbereich A, organisiert durch PD. T. Welte, Magdeburg, sind die klinischen Projekte zusammengefasst. Primär haben die in diesem Bereich aufgelegten wissenschaftlichen Studien eine Verbesserung von Diagnostik und Therapie bei CAP zum Ziel. Dazu gehören beispielsweise die Evaluierung von Präventionsmaßnahmen (Schaberg et al.), Patientenschulung (Welte et al.), Therapieempfehlungen nach ERS-Standard (Ewig et al.), die Detektion von genetischen Merkmalen bei möglichen Hochrisikopatienten (Schumann et al.) sowie die Untersuchung der Rolle von Chlamydia pneumoniae bei Infektionen der unteren Atemwege (Dalhoff et al.).

Die Studien und Untersuchungen im Forschungsbereich B, organisiert durch Prof. Marre, Ulm, fokussieren auf die Erkennung und Charakterisierung von pathogenen Keimen, die eine CAP induzieren können. Dazu gehören S. pneumoniae, Chlamydia pneumoniae, Mycoplasma pneumoniae, Influenzaviren und RSV. Sie machen zusammen die Mehrzahl aller respiratorischen pathogenen Keime aus. Hauptziele sind die Entwicklung, Verbesserung und Evaluierung von diagnostischen Maßnahmen und Werkzeugen. Auch wird der Frage nach „neuen“ Pathogenen, die eine Pneumonie verursachen, nachgegangen vor dem Hintergrund, dass nur in ca. 50\% der Fälle das kausale Pathogen identifiziert werden kann. Dabei werden mikrobiologische Zentren in der gesamten Bundesrepublik zwar unterschiedliche Projekte vorantreiben, die Daten aber werden in einer zentralen Datenbank zusammengeführt und weiter aufbereitet.

Im Projektbereich C, organisiert von Prof. Suttorp, Berlin, ist die Grundlagenforschung gebündelt. Das Hauptaugenmerk gilt der Forschung auf unterschiedlichen Komplexitätsniveaus. Die Experimente in diesem Bereich sollen dazu beitragen, ein besseres Verständnis über die pathogenen Keime und die Wirt-Keim-Interaktionen zu entwickeln. Molekular- und zellbiologische Experimente, BAL-Analysen, die Untersuchung von Gewebeproben sowie die Arbeit mit transgenen Mäusen werden hier von Experten unterschiedlichster Fachrichtungen vorangebracht. Der Publikation der Ergebnisse auf Kongressen und in hoch angesiedel- ten wissenschaftlichen Journalen wird dabei höchste Priorität beigemessen. Der Projektbereich ist wie kaum ein anderer in alle Strukturen von CAPNetz integriert. So kommen beispielsweise aus den klinischen Zentren die Gewebeproben und die BAL-Samples, aus dem Projektbereich B bestimmte Bakterienstämme, aus der Zentralen Serviceeinheit Patientendaten zu den pathogenen Keimen und aus den Core Facilites die genetisch veränderten Nagetiere (Abb. 5).

Projektbereich D beinhaltet wissenschaftliche Fragestellungen, die mit Drittmitteln aus der pharmazeutischen Industrie oder von Diagnostikaherstellern gefördert werden. Es handelt sich dabei um multizentrische klinische Studien, die in diesem Umfang und unter den vorgegebenen engen Zeitfenstern nur von einem gut funktionierenden Netzwerk suffizient bewerkstelligt werden können. So soll unter anderem die Wirksamkeit verschiedener Antibiotikaregime bei Lungenabszessen und Aspirationspneumonien miteinander verglichen werden. Ein zweites Projekt vergleicht die Wirkung einer 5-tägigen Antibiose mit der einer 10 Tage dauernden bei hospitalisierten und ambulanten Patienten.

Studienschwerpunkte der Z-Projekte sind zum einen die Entwicklung und Analyse von netzwerkumfassenden Fragestellungen zur Epidemiologie und Versorgungssituation der CAP sowie epidemiologische und statistische Unterstützung von Teilprojekten (Raspe, Schäfer et al.) und zum anderen Aufbau und Unterstützung in der Informationstechnologie für das gesamte CAPNetzwerk (Weber et al.).

\section{Kommunikation im Netzwerk}

Ein Netzwerk, dessen Mitglieder, klinische Zentren, Forschungslabors und Verwaltungseinrichtungen über ganz Deutschland verstreut sind, braucht als unabdingbare Grundvoraussetzung eine professionelle Kommunikationskultur und -struktur. Die lokalen klinischen Zentren - bestehend aus Kliniken, niedergelassenen Praxen und Labors sowie den Forschungsprojekten in den Bereichen A bis D - sind als „Netzwerk im Netz“ definiert. Ein Lokaler Persönlicher Tutor (LPT) fungiert in den einzelnen Zentren

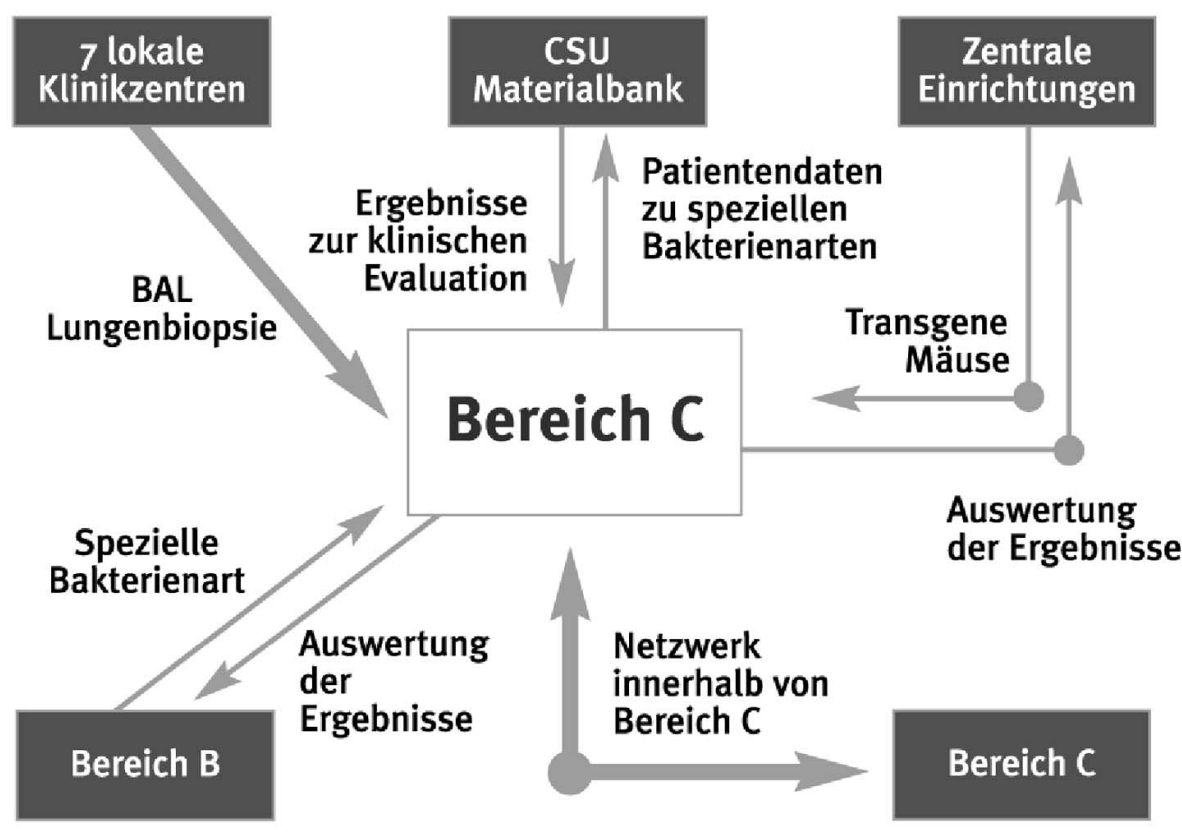

Abb. 5 Integration der Area C. 
als Kommunikationsmanager, der aktiv auf die Teilnehmer zugeht und die Kommunikation innerhalb des Netzwerkes fördert. Ausgebildet und vorbereitet auf ihre Managementaufgabe werden die LPTs von CAPNetz. Wegen der besonderen Verantwortung, die die Position beinhaltet, wird jeder einzelne LPT vom Sprecher von CAPNetz sowie dem lokalen klinischen Zentrum gemeinsam ausgewählt und ernannt. Die LPTs, die für die Kommunikation der Netzwerkmitglieder untereinander ebenso verantwortlich sind wie für die Organisation von lokalen Workshops sowie den Daten- und Probentransfer an die zentralen Labor- und Verwaltungseinrichtungen, sind via Internet sowohl mit der Zentralen Serviceeinheit als auch untereinander in engem Kontakt. Videokonferenzen und Chat-Rooms sollen die flächendeckende Kommunikation erleichtern.

Neben dieser kontinuierlichen Kommunikation wird CAPNetz auch mindestens ein jährliches wissenschaftliches Symposium als Plattform für den Austausch neuester Forschungs- und Studienergebnisse anbieten. Den Wissenschaftlern aus dem Bereich Grundlagenforschung (Projektbereich C) wird eine Hauptaufgabe zukommen bei der Organisation und Durchführung dieses Symposiums, das vorzugsweise im Rahmen des Jahreskongresses der Deutschen Gesellschaft für Pneumologie stattfinden soll. Mit diesem Symposium soll versucht werden, die natürlich unterschiedlich ausgeprägten Interessen und Arbeitsschwerpunkte von Klinikern, Praktikern und Grundlagenforschern auf eine gemeinsame Basis zu stellen.

\section{Transfer des medizinischen Wissens}

Zusätzlich zu Definition und Sicherung von diagnostischen und therapeutischen Standards ist die Weitergabe des medizinischen Wissens ein zentrales Anliegen von CAPNetz. Das Informationsangebot richtet sich dabei nicht nur an Ärzte und medizinisches Assistenzpersonal, sondern beinhaltet auch Patientenaufklärung und -schulung. Die Weitergabe des medizinischen Wissens wird über interaktive Fortbildung mit einem umfassenden Seminarangebot und einem über das Internet erreichbaren Lernprogramm sichergestellt. Dabei wird berücksichtigt, dass Ausgangswissen und Lernziele der Teilnehmer unterschiedlich sind. Videoprogramme ermöglichen die Vorstellung interessanter Erkrankungsgeschichten und -verläufe. Weiterhin stellt CAPNetz einen Expertenrat per Internet zur Verfügung. Unter der E-mail-Adresse expert@capnetz.de können allgemeine und Patienten-bezogene Fragen an das Expertengremium von CAPNetz gestellt werden.

\section{CAPNetz: Die Finanzierung}

Gefördert wird das Kompetenznetzwerk ambulant erworbene Pneumonien durch das Bundesministerium für Bildung und Forschung (BMBF). Zunächst gilt diese Unterstützung für drei Jahre. Kann "CAPNetz“ in dieser Zeit seine Kompetenz unter Beweis stellen, besteht die Möglichkeit zur Anschlussförderung.

\section{CAPNetz sucht weitere Teilnehmer}

Ein essenzielle Aufgabe von CAPNetz ist es, neue Praxen und Labors für die Mitarbeit zu gewinnen und erfolgreich zu integrieren. Vor diesem Hintergrund versteht sich CAPNetz für seine
„Mitglieder“ auch nicht als Aufsichtsorgan, sondern als Mentor, der alle erdenkliche Unterstützung anbietet, um die wissenschaftlichen Projekte so effizient wie möglich voranzubringen. In einem Kooperationsvertrag werden die Maßnahmen, die Rechte und Pflichten für beide Seiten klar niedergeschrieben. Die Mitarbeit in CAPNetz steht prinzipiell allen niedergelassenen Ärzten, Kliniken und Ambulanzen offen, die ein tieferes Interesse an pneumologischen Fragestellungen haben und die Diagnostik und Therapie im Rahmen der klinischen Pneumologie anbieten und durchführen. Spezielle Zertifizierungen durch CAPNetz weisen die teilnehmenden Ärztinnen und Ärzte zudem als besonders geschult auf dem Gebiet der Diagnostik und Therapie von ambulant erworbenen Pneumonien aus. Die Teilnehmer am CAPNetz sind jedoch gehalten, bei ihrer wissenschaftlichen Arbeit strikt die Vorgaben von GLP, GCP und GEP zu beachten. Nur so kann ein hoher und international anerkannter Qualitätsstandard vom ersten bis zum letzten Patienten, von der ersten Probenentnahme bis zur abschließenden Publikation der Ergebnisse erreicht werden.

Die Einhaltung internationaler Standards ist auch deshalb geboten, weil sich CAPNetz im Zuge des 6. EU-Rahmenprogramms für Forschung und technologische Entwicklung (FTE) um die Etablierung eines europäischen Netzwerkes zur CAP bemüht.

Weitere Informationen sind abrufbar über die Homepage des Netzwerkes: www.capnetz.de

Weitere Informationen können zudem angefordert werden über: CAPNetz-Sprecher

Prof. Dr. Norbert Suttorp

Med. Klinik/Infektiologie

Charité, Augustenburger Platz 1

13353 Berlin

Telefon: $030-450-553051$

oder

\section{Geschäftsstelle CAPNetz}

Universitätsklinik Ulm

Albert-Einstein-Allee 47

$89069 \mathrm{Ulm}$

Tel. $0731-50025303$

Fax 0731-500 25339

\section{Zusammenfassung}

Die ambulant erworbene Pneumonie (CAP) ist eine Volkskrankheit, die häufiger zur stationären Aufnahme führt als Schlaganfall oder Herzinfarkt. Die erzielten diagnostischen und therapeutischen Fortschritte resultierten leider nicht in einer verbesserten Versorgung von Pneumoniepatienten. Das vom BMBF geförderte Kompetenznetzwerk CAPNetz strebt an, durch eine engere Verzahnung des ambulanten und stationären Bereichs auf der einen und durch bessere Koordination der Forschung hinsichtlich Versorgung, Klinik und Grundlagen Fortschritte in der Überwindung dieser Erkrankung zu erzielen. Zum ersten Male seit 20 Jahren werden von öffentlicher Seite Untersuchungen zu Pneumonie gefördert. Nutzen wir diese Chance! 
Projekte im Kompetenznetz „Ambulant erworbene Pneumonie“: CAPNetz

A1 PD Dr. med. Tobias Welte Prof. Dr. B. P. Robra

A2 PD Dr. med. Santiago Ewig PD Dr. med. Gerd Fätkenheue

A6 Prof. Dr. med. Klaus Dalhoff

B1 PD Dr. Michael Wagner PD Dr. med. Matthias Horn Prof. Karl-Heinz Schleifer

B2 Prof. Dr. med. habil. Eberhard Straube PD Dr. med. Heike Freidank Prof. Dr. med. Matthias Maass PD Dr. med. Andreas Essig

B3 Dr. med. Christian Lück Prof. Dr. med. Enno Jacobs

B4 PD Dr. med. Ralf René Reinert

2 Prof. Dr. Stefan H.E. Kaufmann

Prof. Dr. med. Jürgen Lohmeyer

C3 Prof. Dr. med. Jürgen Lohmeyer

C4 Frau. Prof. Simone Rosseau Prof. Dr.med. Norbert Suttorp

C5 Prof. Dr. med. Ralf Schumann

C6 Dr.rer.nat. Jürgen Rödel Prof. Dr. med. habil. Eberhard Straube

C8 Dr. Sven Hammerschmidt Prof. Dr. G. Singh Chhatwal

C10 Prof. Dr. Reinhard Marre

D1 Prof. Dr. med. Hartmut Lode

$Z 1$ Dr. med. Klaus Richter

Z2 Prof. Dr. med. Dr. phil. Heiner Raspe Prof. Dr. med. Torsten Schäfer

Z3 Prof. Dr. Michael Weber

Z4 Prof. Dr. Reinhard Marre
Epidemiology of community acquired pneumonia (CAP) in outpatients. Impact of an educational program on hospitalization rate and outcome

A controlled study of the impact of

the implementation of ERS-guidelines on clinical outcomes of patients with community acquired pneumonia (CAP)

Chlamydia pneumoniae and lower respiratory tract infections (LRTI): Persistence and triggering of inflammatory airway diseases

Detection and in situ identification of members of the Chlamydiaceae and Parachlamydiaceae as pulmonary pathogens. Are Parachlamydiaceae new emerging pathogens?

Standardisation and evaluation of microbiological diagnosis of community acquired pneumonia caused by

Chlamydia pneumoniae

Diagnosis of respiratory infections caused by Legionellae spp. or Mycoplasma pneumoniae by new molecular approaches

Molecular characterization of S. pneumoniae isolated from patients with CAP in Germany

Detection and molecular characterization of influenza viruses and respiratory syncytial virus as agents for acute respiratory diseases and pneumonia

Role of Regional Cytokine Production in Pneumonia

Triggering alveolar monocyte recruitment and activation in mice via bacterial pattern recognition receptor (PRR) ligands: role in lung injury and host defense

Host defense mechanisms in the lung Impact of Interleukin-15 and role of apoptosis

S. pneumoniae induced signal transduction: role of toll-like receptors (TLR-2 and -4), MD-2 and LPS binding protein (LBP) and implications for pathogenesis of pneumonia Airway remodeling and Chlamydia pneumoniae

Comparison of protein expression profiles of pneumococcal carrier and invasive strains

Role of regulatory genes of Legionella pneumophila in virulence

Clinical trials

Administration

Epidemioloy, Statistics, Health Services Research

Electronical communication and database

Core facility for strain and specimen
${ }^{1}$ Osler W. Lobar pneumonia. In: The principles and practice of of medicine. Edinburgh \& London: Young J, Pentland, 1901: 126-129

${ }^{2}$ Chanock RM, Dienes L, Eaton MD. Mycoplasma pneumoniae: proposed nomenclature for atypical pneumonia organism. Science 1963; 140: $662-669$

${ }^{3}$ Farr BM, Kaiser DL, Harrison BDW et al. Prediction of microbial aetiology at admission to hospital for pneumonia from the presenting clinical features. Thorax 1989; 44: 1031 - 1035

4 Tew J, Calenoff L, Berlin BS. Bacterial or nonbacterial pneumonia: accuracy of radiographic diagnosis. Radiology 1977; 124: 607-612

${ }^{5}$ Woodhead M, Torres A. Definition and classification of communityacquired and nosocomial pneumonias. Eur Respir Mon 1997; 3: 1 - 12

${ }^{6}$ Escamilla R, Hermant C. Pneumonia in immunocompromised patients. Eur Respir Mon 1997; 3: 189-208

7 Pingleton SK, Fagon JY, Leeper KV. Patient selection for clinical investigation of ventilator-associated pneumonia. Chest 1992; 102 (Suppl. 1): 557S-564S

${ }^{8}$ ERS Task Force Report. Guidelines for management of adult community-acquired lower respiratory tract infections. Eur Respir J 1998; 11: $986-991$

${ }^{9}$ Bartlett JG, Breiman RF, Mandell LA et al. Community-acquired pneumonia in adults: Guidelines for management. Clin Infect Dis 1998; 26: $811-838$

${ }^{10}$ Lopez AD, Murray CC. The global burden of disease. Nat Med 1998; 4 : $1241-1243$

11 Center for Disease Control and Prevention. Premature deaths, monthly mortality and monthly physician contacts - United States. MMWR Morb Mortal Wkly Rep 1997; 46: 556

12 Marston BJ, Plouffe JF, File TM et al. Incidence of community acquired pneumonia requiring hospitalizations. Arch Intern Med 1997; 157: 1509 - 1518

${ }^{13}$ Niederman MS, McCombs JS, Unger AN et al. The cost of treating community-acquired pneumonia. Clin Ther 1998; 20: 820-837

14 Fine MJ, Auble TE, Yealy DM et al. A prediction rule to identify low-risk patients with community acquired pneumonia. N Engl J Med 1997; 336: $243-250$

${ }^{15}$ Marrie TJ, Lau CY, Wheeler SL et al. A controlled trial of a critical pathway for treatment of community-acquired pneumonia. JAMA 2000; 283: $749-755$

${ }^{16}$ Fine MJ, Smith MA, Carson CA et al. Prognosis and outcome of patients with community acquired pneumonia. JAMA 1996; 275: 134-141

17 Dean NC, Silver MP, Bateman KA et al. Decreased mortality after implementation of a treatment guideline for community-acquired pneumonia. Am J Med 2001; 110: 451 - 457

18 Moroney JF, Fiore AE, Harrison LH et al. Clinical outcomes of bacteremic pneumococcal pneumonia in the era of antibiotic resistance. Clin Infect Dis 2001; 33: 797-805

${ }^{19}$ Reinert RR, Al-Lahham A, Lemperle M et al. Emergence of macrolide and penicillin resistance among invasive pneumococcal isolates in Germany. J Antimicrob Chemother 2002; 49: 61 - 68

${ }^{20}$ Priest P, Yudkin P, McNulty C. Antibacterial prescribing and antibacterial resistance in English general practice: cross sectional study. BM] 2001; 323: 1037 - 1041

${ }^{21}$ Ross JJ, Worthington MG, Gorbach SL. Resistance to levofloxacin and failure of treatment of pneumococcal pneumonia. N Engl J Med 2002; 347: $65-67$

${ }^{22}$ Marrie TJ, Peeling RW, Fine MJ. Ambulatory patients with community acquired pneumonia, the frequency of atypical agents and clinical course. Am J Med 1996; 101: 508 - 515 